\title{
AFM observation of heightened cell periphery of high-grade glioblastoma cell lines
}

\author{
James R. Smith*, Zaynah Maherally, Samantha C. Higgins, Qian An, Helen L. Fillmore
} and Geoffrey J. Pilkington

Cellular and Molecular Neuro-oncology Research Group, School of Pharmacy and Biomedical Science, University of Portsmouth, St Michael's Building, White Swan Road, Portsmouth PO1 2DT, UK.

*Corresponding author: Tel: +44 (0)23 9284 2556; Fax: +44 (0)23 9284 3565;

Email: james.smith@port.ac.uk

\begin{abstract}
Glioblastoma multiforme (GBM) is a highly invasive (WHO grade IV) brain tumour that has a very poor prognosis for patients with the condition (median survival 14.2 months). Quantitative Imaging (QI) mode $\circledR$ atomic force microscopy (AFM) was used to measure the heights of the leading-edge cell peripheries, the lamellipodia, of two such cell lines (SNB-19 and UP-007), together with those from non-neoplastic astrocyte control cells (CC-2565 and SC-1800) and from a low-grade (WHO grade I) glioma cell line (SEBTA048). The lamellipodia heights of the glioma cells SNB-19 and UP-007 were $2.45 \pm 0.59$ and $1.57 \pm 0.42 \mu \mathrm{m}$, respectively, which were higher than those of the CC-2565 and SC1800 cells $(1.03 \pm 0.58$ and $0.85 \pm 0.40 \mu \mathrm{m}$, respectively; $p<0.0001$, except between CC-2565 and UP-007, $p<0.001)$. Lamellipodia height differences between the two glioma cell lines $(p<0.0001)$, might be attributed to the measured difference in invasive potential between these two cell lines. The equivalent lamellipodia height of the SEBTA048 cells was $1.16 \pm 0.48 \mu \mathrm{m}$, the same as that of the astrocytes $(p>0.05)$, but lower than those of the high-grade gliomas ( $p<0.0001$ and $p<0.01$ for SNB-19 and UP-007, respectively). These measured heights, therefore, may provide new insights for monitoring and controlling cellular invasion in brain tumours.
\end{abstract}

Keywords: Glioblastoma multiforme (GBM), brain tumour, invasion, cytoskeleton, lamellipodia, atomic force microscopy (AFM).

\section{Introduction}

Glioblastoma multiforme (GBM) is a heterogeneous and highly invasive WHO grade IV [1] brain tumour that leads to a poor prognosis for patients (median survival 14.2 months, 2-year survival $26 \%$, with surgery and radiotherapy with concomitant or adjuvant chemotherapy) [2].

The use of atomic force microscopy (AFM) nanoindentation measurements to study stiffness of cancer cells has received much attention [3-6]. We recently investigated the expression of cytoskeletal proteins and cellular stiffness of glioma cells (SNB-19), with and without siRNA knock down CD44, a cell adhesion molecule (CAM) long associated with gliomas [7], and also compared these to non-neoplastic astrocytes (CC-2565, control) [8]. Silencing CD44 SNB-19 led to changes in cytoskeletal protein expression, making them less migratory, and AFM showed a reduction in Young's Modulus $(E=0.56$ 
$\pm 0.50 \mathrm{kPa}$, nuclear regions) relative to the parental cells $(E=1.93 \pm 2.86 \mathrm{kPa} ; p<$ $0.001)$. We hypothesised that CD44 signalling via cytoskeletal proteins such as vimentin may influence the ability of glioma cells to respond to host-tumour derived mechanical pressures.

In this paper, we report on the surprisingly large heights along portions of the cell periphery leading edge, the lamellipodia [9], of GBM cells from two cell lines, SNB-19 and UP-007. Lamellipodia are Arp2/3 complex-dependent, sheet-like membrane protrusions at the leading edge of migrating cells that contain a dense network of branched actin filaments $[10,11]$. For comparisons, we have also examined the peripheries of cells from a low-grade, WHO grade I [1], dysembryoplastic neuroepithelial tumour (DNET) glioma cell line (SEBTA-048) and from two non-neoplastic astrocyte cell lines (CC-2565 and SC-1800). Here, we hypothesise that the heightened features of the lamellipodia might provide a new indicator of cellular invasion. In a recent paper, 'dynasore', a dynamin inhibitor, was found to suppress lamellipodia formation and invasion of U2OS human osteosarcoma cells [12]. Thus, the lamellipodia might provide an attractive target to monitor and control cellular invasion and migration in brain tumours.

We have used AFM Quantitative Imaging (QI) mode ${ }^{\circ}$ (JPK Instruments), a fast force mapping technique [13], to image the cells. This technique allows the height at which the tip, measured from each approach force curve (an array of curves constitutes each map), just makes contact with the sample (set-point $=0 \mathrm{nN}$ ). These data were subsequently used to produce contact point ('zero force') topography images. It was thought that this technique would lead to significantly less compression of delicate lamellipodia structures than with standard Tapping Mode AFM imaging. Light microscopy, including live cell imaging, and an invasion assay were used as supporting techniques.

\section{Materials and methods}

\subsection{Cell culture}

Non-neoplastic foetal cerebral astrocytes (CC-2565), passage-5, (Lonza, UK) and human normal astrocytes (SC-1800), passage-6, derived from the cerebral cortex (ScienCell, USA) were grown in astrocytes basal media (AGM) (Lonza, Germany) supplemented with SingleQuots (Lonza, Germany) and 3\% human serum (Sigma, UK). The established human glioblastoma cell line, SNB-19 (passage-44), was obtained from the DSMZ German Brain Tumour Bank (Germany). Human glioblastoma cell line, UP-007 (passage12) and low grade glioma (WHO grade I) cell line SEBTA-048 (right temporal lobe; passage-4) were established in-house from biopsy-derived cultures. All glioma cells were cultured in Dulbecco's modified Eagle medium (DMEM; Fisher, UK) supplemented with $10 \%$ human serum (Sigma, UK) and maintained at $5 \% \mathrm{CO}_{2}$ and humidified atmosphere at $37^{\circ} \mathrm{C}$. All cell lines were subjected to routine mycoplasma testing utilising a kit from Lonza. Cell authentication was conducted using a microfluidic electrophoresis system incorporating an Agilent 2100 Bioanalyzer (Agilent Technologies, USA), to analyse STRPCR fragments from 10 human genomic loci of human cell lines [14].

\subsection{Live cell imaging microscopy and phase contrast microscopy}

Cells of interest were plated at $30 \%$ confluency in a 24 -well plate and left to adhere overnight prior to each live cell experiment. Live cell imaging was performed using a Zeiss Axiovert 200M (inverted) microscope (Carl Zeiss, Welwyn Garden City, Herts, UK) contained in an incubator $\left(37{ }^{\circ} \mathrm{C}, 5 \% \mathrm{CO}_{2}\right.$, humid atmosphere). A $40 \times$ objective was used and phase images were consecutively acquired typically at 5 frames $\mathrm{s}^{-1}$ for $60 \mathrm{~s}$ (Volocity software V 6.1.1, Perkin Elmer, Waltham, MA, US). Phase images were 
subsequently extracted from live cell movie sequences. Lower magnification phase contrast images (20× objective) were also acquired using an Olympus IX71 inverted microscope (Shinjuku, Tokyo, Japan); contrast and brightness were adjusted slightly in MicroSoft PowerPoint.

\subsection{Invasion}

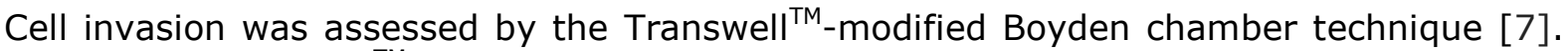
Briefly, the Transwell ${ }^{\mathrm{TM}}$ inserts used were $8 \mu \mathrm{m}$ porosity, polycarbonate membrane filters contained within 24-well plates (Corning, UK) and were coated with BD Matrigel ${ }^{\mathrm{TM}}$ (50 $\mathrm{\mu l}$, $0.5 \mathrm{mg} / \mathrm{ml}$; Scientific Laboratory Supplies, Nottingham, UK). Platelet-derived growth factor $\left(\mathrm{PDGF}_{\mathrm{AB}} ; 10 \mathrm{ng} / \mathrm{ml}\right)$ was used as a chemoattractant in the lower compartment. Invasion was allowed to occur for $6 \mathrm{~h}$ in an incubator $\left(37{ }^{\circ} \mathrm{C}, 5 \% \mathrm{CO}_{2}\right)$. A time-point analysis ( $2 \mathrm{~h}, 4 \mathrm{~h}, 6 \mathrm{~h}, 8 \mathrm{~h}, 10 \mathrm{~h}, 12 \mathrm{~h}$ ) was carried out before choosing ' $6 \mathrm{~h}$ ' as the optimal incubation time for the invasion assay, which was further supported by previous studies [15]. This provided enough time for SNB-19 and UP-007 cells to invade prior to proliferation based on the population doubling times calculated for these cell lines. The filters were then removed and fixed with $4 \%$ paraformaldehyde (PFA). Non-invading cells on the upper surface of the filter were removed and invaded cells, adherent on the lower filter surface, were characterised through alkaline phosphatase vector red (Vectorlabs, UK) staining. Cells positive for CD44 were thus stained red. Nuclei were blue counterstained with Haematoxylin (Dako, UK). Images were captured with a Zeiss Axiophot brightfield microscope using the AxioVision software (V4.4, Carl Zeiss) with a $20 \times$ objective. Cells were counted in 5 random fields, with each experiment being performed in triplicate.

\subsection{AFM}

AFM measurements were performed using a NanoWizard 3 AFM (JPK Instruments, Berlin, Germany) mounted on a Zeiss Axiovert 200M (Carl Zeiss) inverted optical microscope placed on a Halcyonics Micro 40 anti-vibration table (Accurion, Goettingen, Germany). JPK AFM image analysis software (V 5.1.13) was used for image acquisition and analysis. The serum growth media from a Petri dish (glass-bottomed) of cells of interest was removed (pipette) and replaced with 'imaging buffer' (DMEM/F-12 nutrient mixture (Ham) (1:1) (1X); Gibco, Life Technologies, Paisley, UK). The Petri dish (lid removed) was then placed on an integrated Petri dish heater $\left(37^{\circ} \mathrm{C}\right.$; JPK Instruments) and a $20 \times$ objective, on the inverted microscope, was used to locate cells of interest. An 'arrowshaped' Si cantilever (HQ:CSC37/No Al; probe ' $\mathrm{C}^{\prime}$, nominal length $I_{\text {nom }}=300 \pm 5 \mu \mathrm{m}$, width $w_{\text {nom }}=35 \pm 3 \mu \mathrm{m}$, thickness $t_{\text {nom }}=2.0 \pm 0.5 \mu \mathrm{m}$, spring constant $k_{\text {nom }}=0.4 \mathrm{~N} \mathrm{~m}^{-}$ 1; Mikromasch Europe, Wetzlar, Germany) with integrated tip ( $R=8 \mathrm{~nm}, h=12-18$ $\mu \mathrm{m}$ ) was used for all measurements. The actual $k$ value was measured as $0.2180 \mathrm{~N} \mathrm{~m}^{-1}$ prior to imaging using the method of Hutter and Bechhoefer [16] and obtaining a force $v s$. distance curve on the bottom of the glass Petri dish (in media) in a region devoid of cells. AFM QI mode ${ }^{\circledR}$ images ( $64 \times 64$ arrays of force-distance curves) of located cells were obtained (approach and retract, $z$ length $=5.0 \mu \mathrm{m}$, approach $=$ retract velocity 250 $\mu \mathrm{m} \mathrm{s}^{-1}$, approach $=$ retract time $=20 \mathrm{~ms}$; image acquisition time $=2.88 \mathrm{~min}$, set-point $=6.0 \mathrm{nN}$ ) over a scan range of $100 \mu \mathrm{m} \times 100 \mu \mathrm{m}$. AFM contact point images, representing cell topographies in the near-absence of an applied load $(0 \mathrm{nN})$ from the tip/cantilever, were extracted from the approach force curves comprising the QI mode $\mathbb{R}$ images. Height data of cell lamellipodia were extracted from transects drawn across the contact point images ( $n=10$ per cell, 3 or 4 cells per cell line; mean and sd per cell lines were reported). Combined height values, per cell line, were analysed using a one-way ANOVA, with a Tukey post-hoc test $(*, p<0.05 ; * *, p<0.01 ; * * *, p<0.001 ; * * * *, p$ $<0.0001$ ) in SPSS (Version 22; IBM, NY, US). Contact point images were filtered using a median pass filter. 


\section{Results and discussion}

\subsection{Live cell imaging}

Prior to the AFM studies, an overview of the cellular morphology and presence of lamellipodia were investigated. Lamellipodia were present at the leading edge of a number of migrating cells, as confirmed by live cell imaging (those of UP-007 cells are shown in Fig. 1). These structures could be seen as bright-shaded, ruffled edges on generally semi-circular protruded regions.
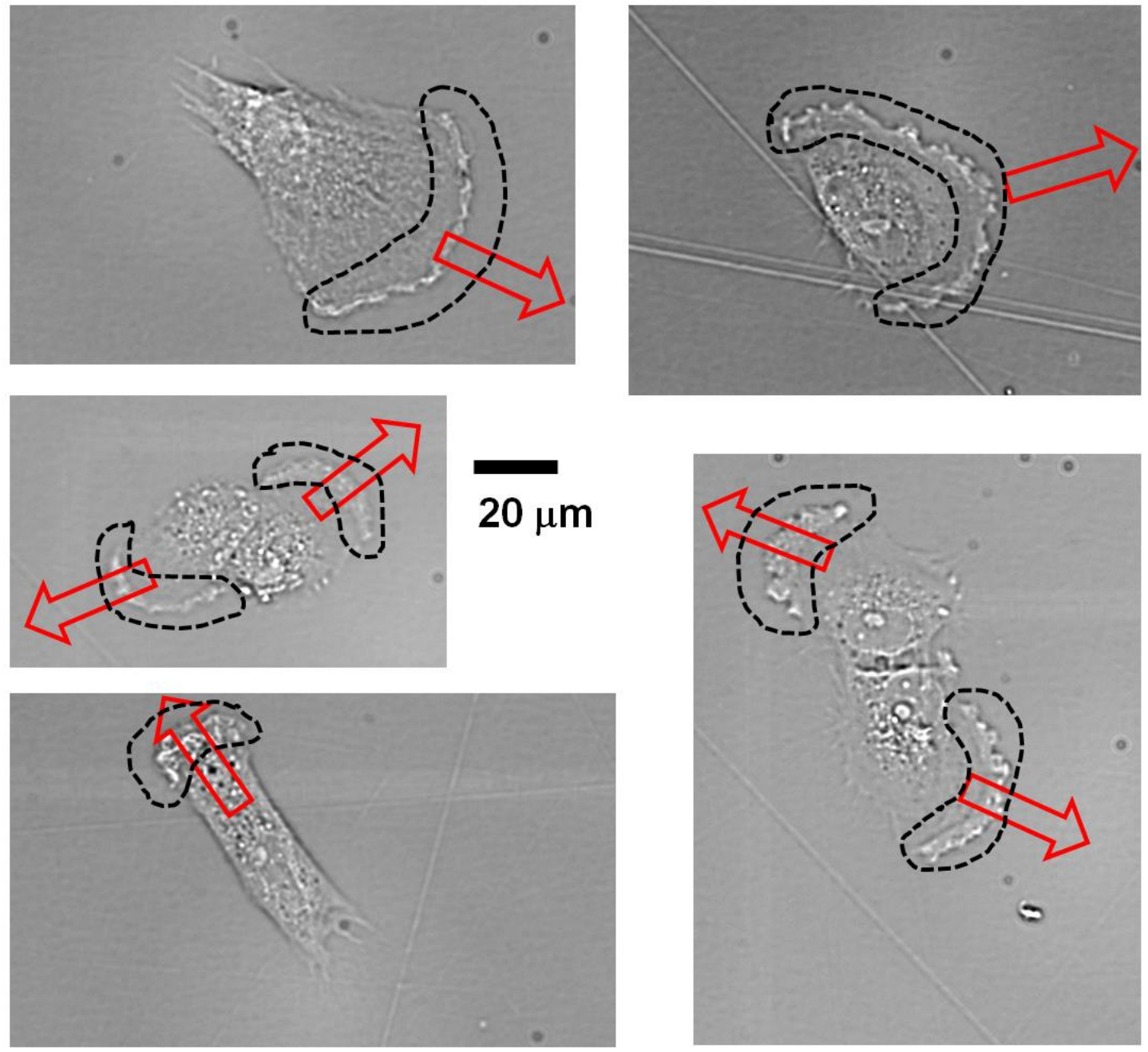

Fig. 1. Light microscopy phase contrast images of GBM cells (UP-007) extracted from live cell imaging studies. Pronounced lamellipodia structures can be seen as bright-shaded, ruffled regions at leading edges of moving cells (dotted areas); red arrows show direction of cell movement; $40 \times$ objective, scale bar $=20 \mu \mathrm{m}$.

\subsection{Light microscopy}

CC-2565 cells at $80 \%$ confluence showed elongated and stellate morphology (Fig. 2a). Lamellipodia could be observed at the leading edge of a number of these cells. SC-1800 cells at $60 \%$ confluence showed a polygonal morphology with thin, elongated 
intercommunicating processes at either end of the cells (Fig. 2b). Lamellipodia of similar appearance to those of the CC-2565 cells were observed. SNB-19 cells, at 30\% confluence, showed small, predominantly stellate morphologies, with some flattened, irregular shapes with tiny, slender processes (Fig. 3a). This cell line had the greatest number of lamellipodia, possibly indicative of higher motility and/or invasion [12]. UP007 cells, at $40 \%$ confluence, revealed their heterogeneous nature, with many processes at their ends and with some short, more rounded morphologies with predominantly fusiform cells (Fig. 3b). Similar lamellipodia structures were observed, although to a much reduced frequently compared to their SNB-19 counterparts. The low-grade SEBTA048 cell line, at $70 \%$ confluence, showed rather fat, end-feet processes; again, lamellipodia of similar appearance to those seen above were present (Fig. 4).

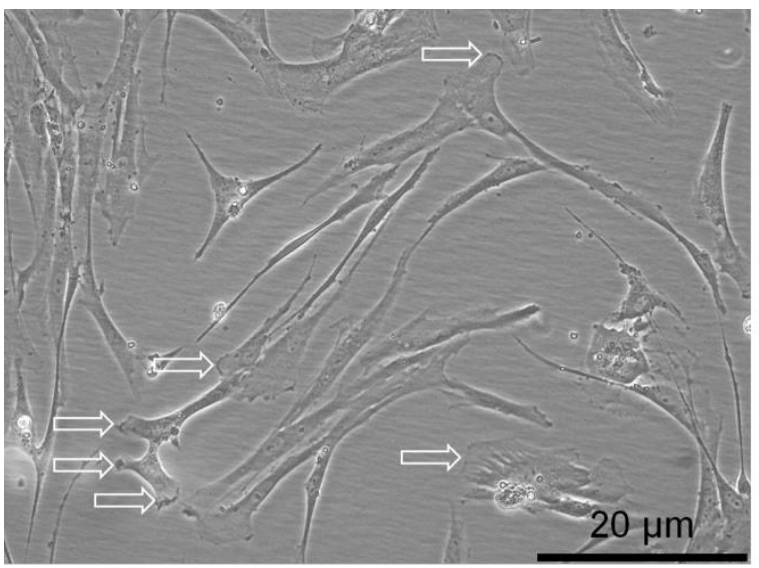

(a)

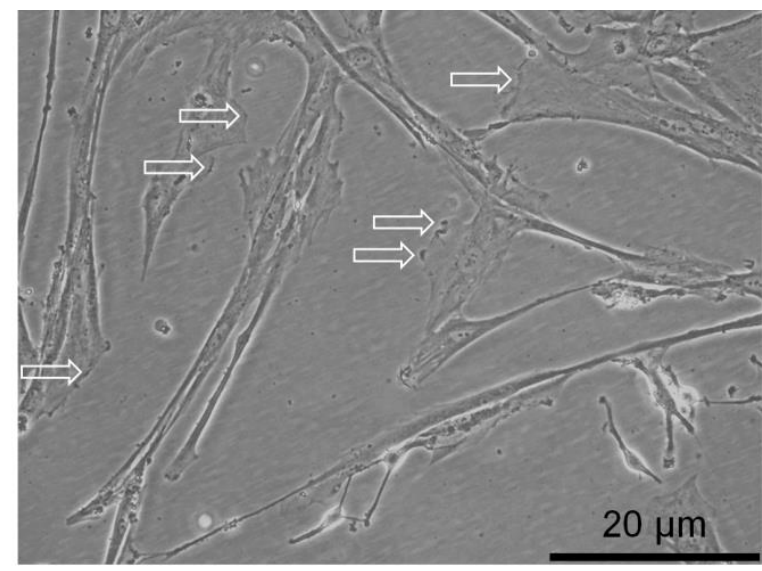

(b)

Fig. 2. Phase contrast micrographs showing morphology of non-neoplastic astrocytes: (a) CC-2565 and (b) SC-1800 cells. Arrows indicate location of lamellipodia. Scale bar $=20 \mu \mathrm{m}$.

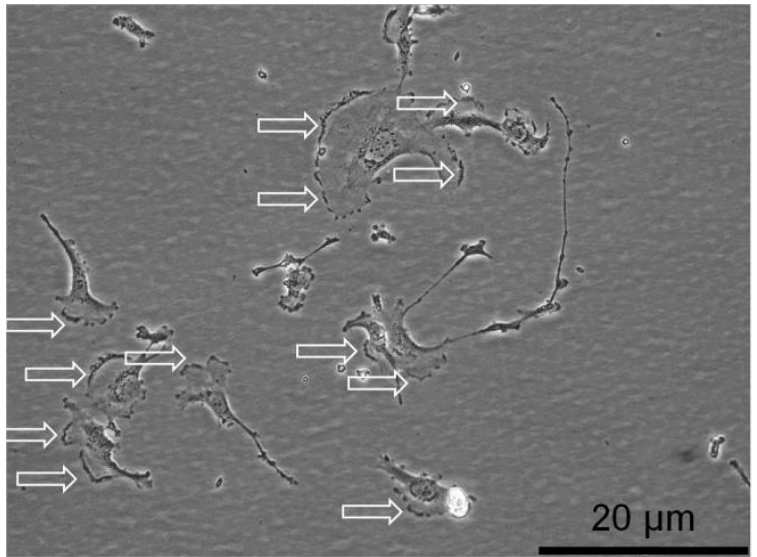

(a)

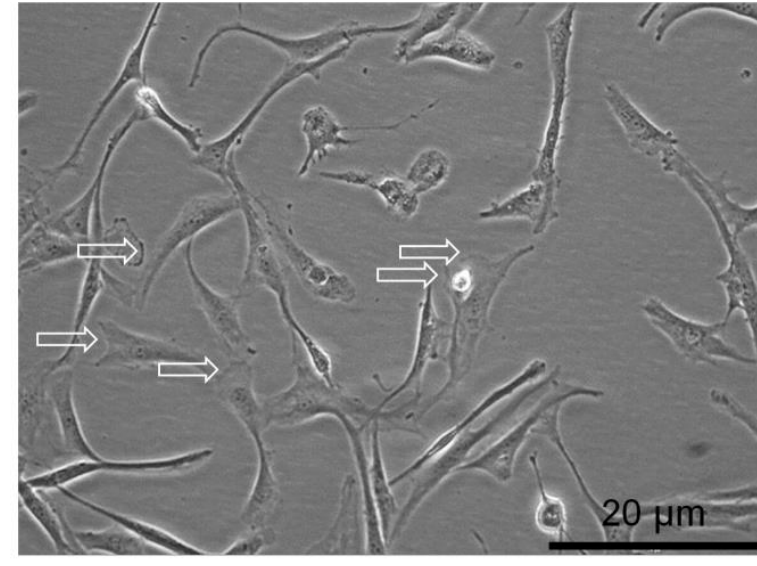

(b)

Fig. 3. Phase contrast micrographs showing morphology of GBM cells: (a) SNB-19 and (b) UP-007 cells. Arrows indicate location of lamellipodia. Scale bar $=20 \mu \mathrm{m}$. 


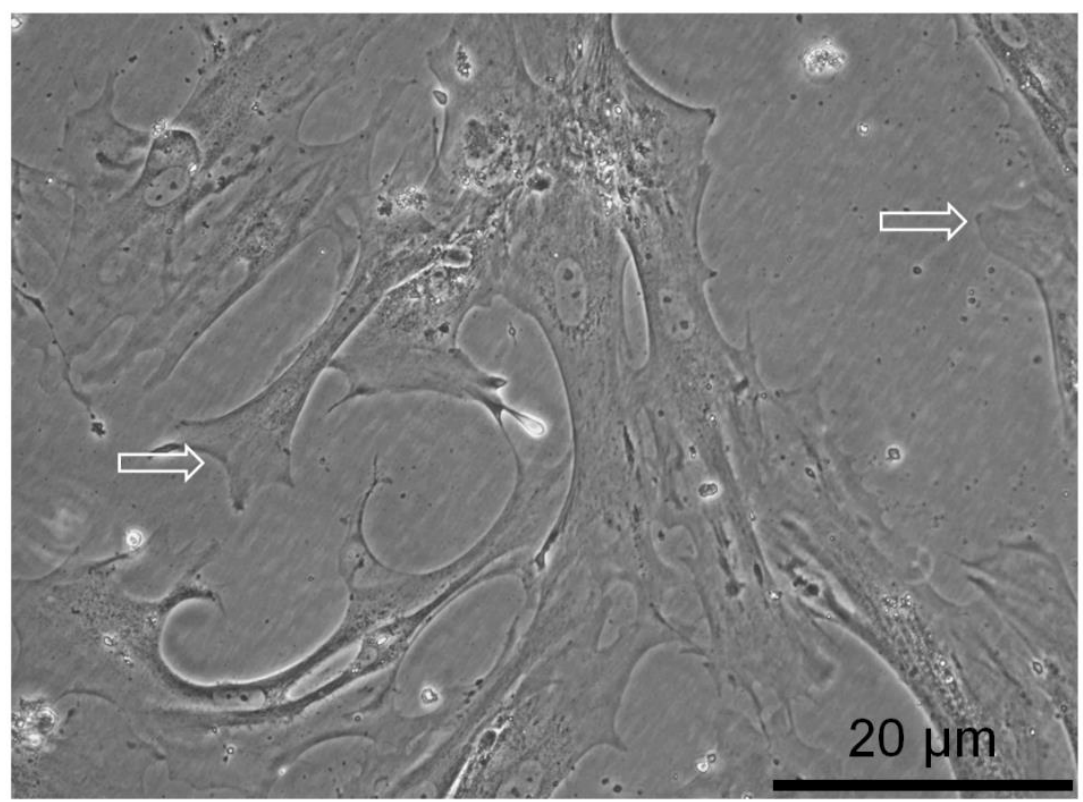

Fig. 4. Phase contrast micrograph showing morphology of low grade glioma (SEBTA-048) cells. Arrows indicate location of lamellipodia. Scale bar $=20 \mu \mathrm{m}$.

\subsection{Invasion}

To investigate whether the difference in number of lamellipodia observed in SNB-19 and UP-007 cells might be indicative of their invasive potential, an invasion assay was performed. The findings showed more SNB-19 cells positive for CD44 (red) had invaded to the lower side of the membrane compared to UP-007 (Fig. 5). SNB-19 cells showed a homogenous morphology with shorter fat processes. These results were reflected in the quantitative analysis, showing SNB-19 cells to be more invasive than those of UP-007 ( $p$ $<0.05$; Fig. 6).

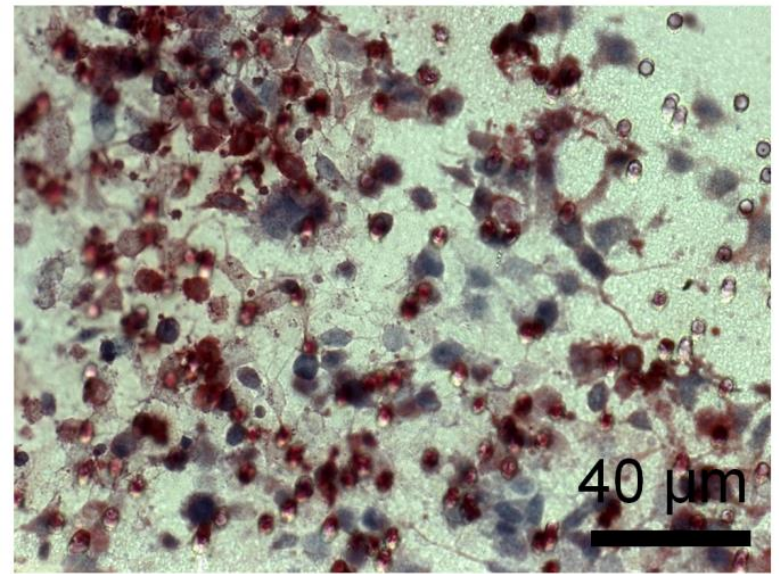

(a)

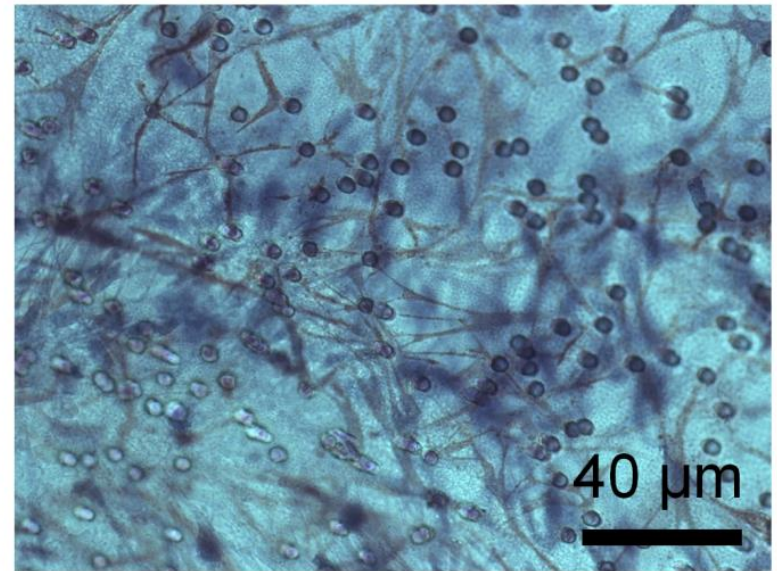

(b) 
Fig. 5. Brightfield micrographs indicative of invasive potential of CD44-positive cells of (a) SNB-19

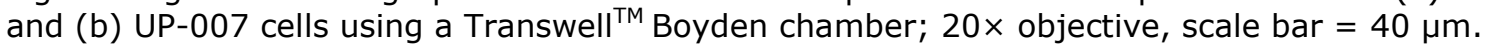

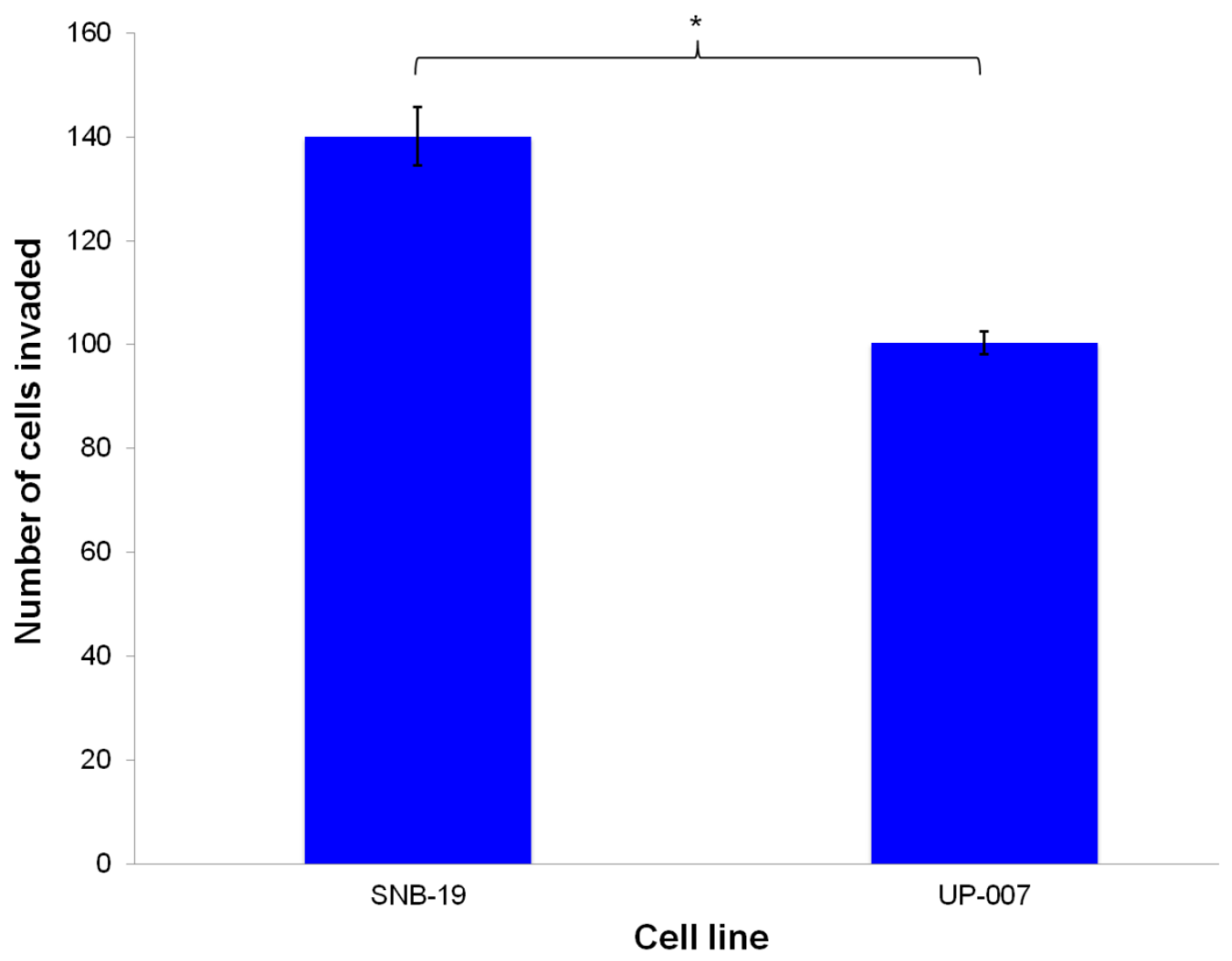

Fig. 6. Number of SNB-19 and UP-007 cells that had invaded through the Transwell ${ }^{\mathrm{TM}}$ inserts. SNB19 cells were significantly more invasive than UP-007 cells $(*, p<0.05)$. The graph is representative of the results obtained by repeating the study three times in triplicate; number of SNB-19 cells invaded $=140$, number of UP-007 cells invaded $=100$.

\subsection{AFM measurements}

Cells for AFM imaging were selected, using the inverted microscope, with the following criteria: that they were not joined to other cells, they exhibited lamellipodia and were free from obvious artefacts. Phase contrast images were obtained for each cell imaged, although are not shown. AFM contact point images of non-neoplastic astrocyte cell lines (CC-2565 and SC-1800; Fig. 7) generally revealed rounded cells, with raised nuclei regions but with little or no apparent lamellipodia despite these features being present in the phase contrast images. Transects drawn across the cells, enabled the lamellipodia heights to be calculated (Fig. 8 shows an example). The combined values for CC-2565 and SC-1800 cell lines were $1.03 \pm 0.58$ and $0.85 \pm 0.40 \mu \mathrm{m}$, respectively. In contrast, many of the glioma cells, from both SNB-19 and UP-007 cell lines, had obvious lamellipodia structures present in the AFM contact point images; they were particularly prominent in the SNB-19 cells (Fig. 9). The lamellipodia heights were $2.45 \pm 0.59$ and $1.57 \pm 0.42 \mu \mathrm{m}$, respectively. These heights were also measured for low grade glioma cells (SEBTA-048) to investigate whether they decreased in magnitude (Fig. 10). The lamellipodia heights here were $1.16 \pm 0.48 \mu \mathrm{m}$, more comparable to the astrocytes. 


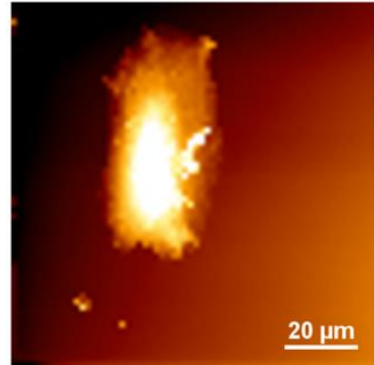

(a)

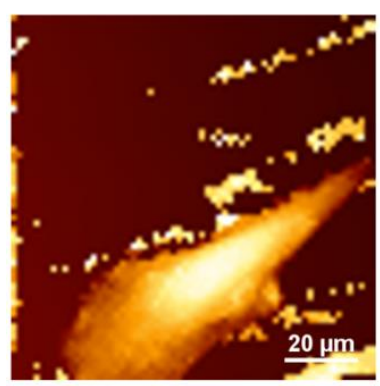

(d)

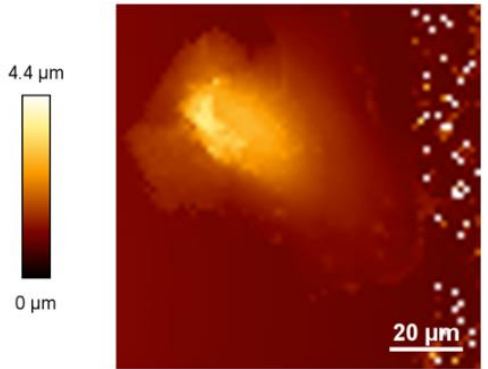

(b)

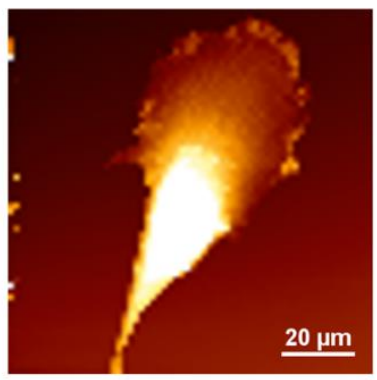

(e)

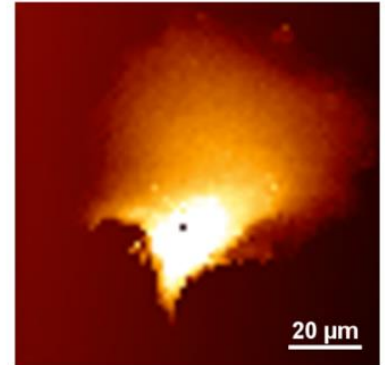

(c)
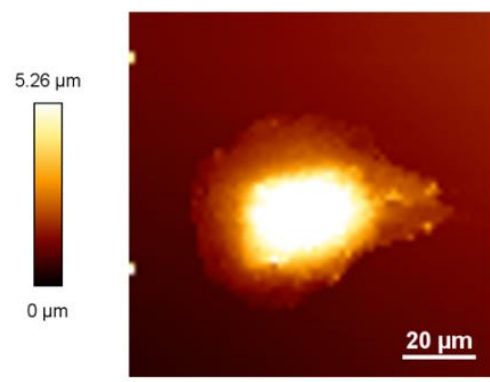

(f)

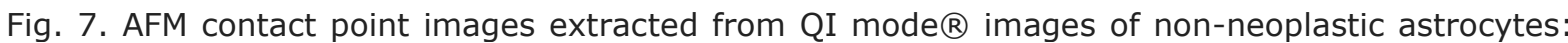
(a-c) CC-2565 cells and (d-f) SC-1800 cells.

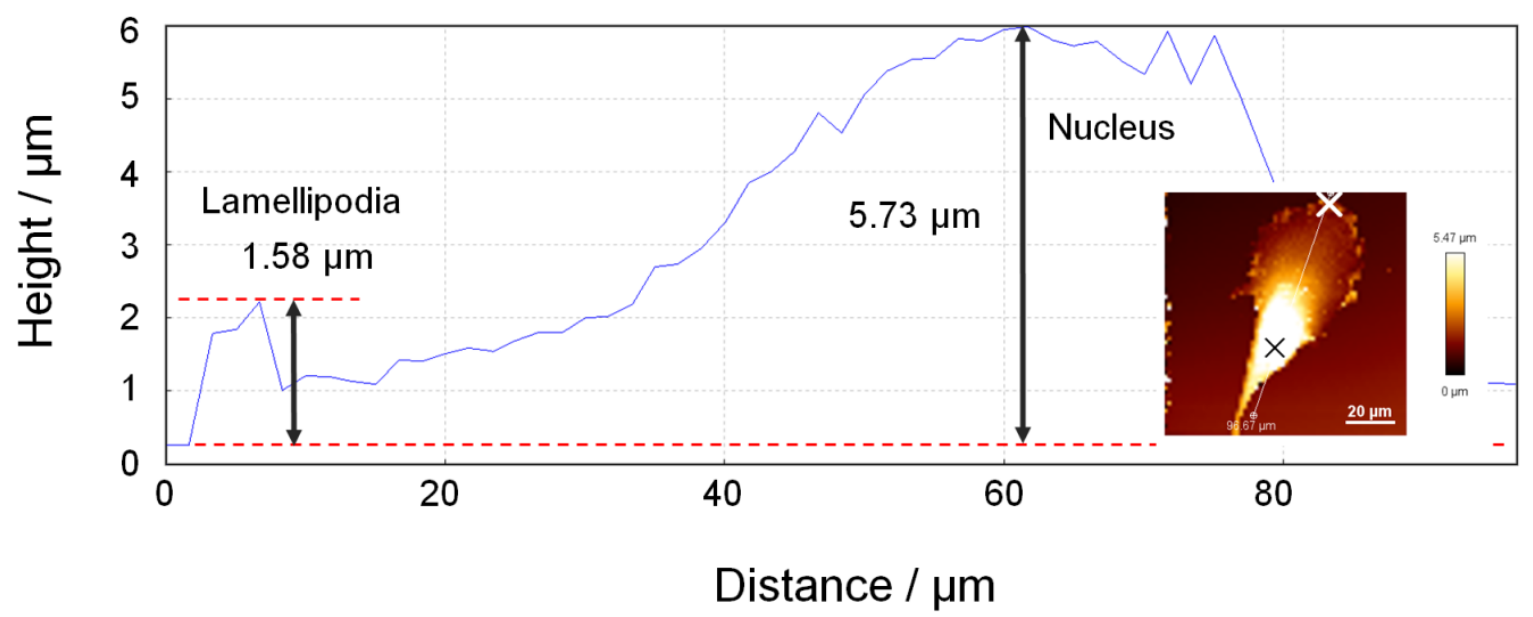

Fig. 8. Transect line denoting location at which lamellipodia height data are extracted from AFM contact point images. The example shows an SC-1800 cell (same as Fig. 7e). 


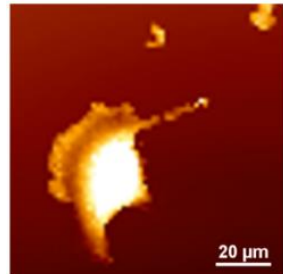

(a)

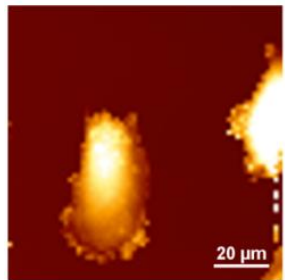

(e)

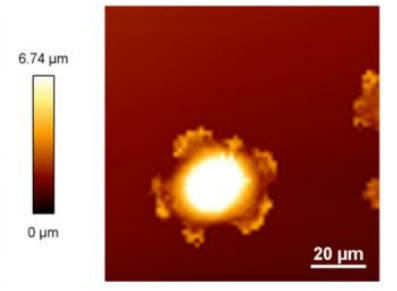

(b)

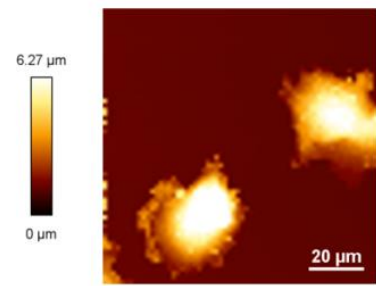

(f)

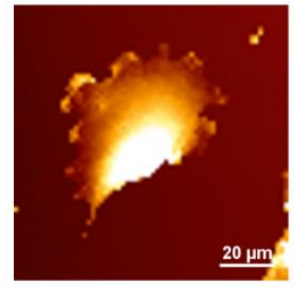

(c)

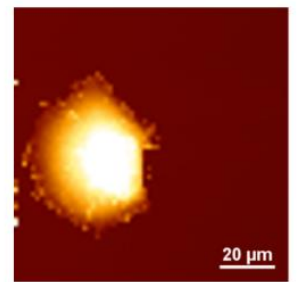

(g)

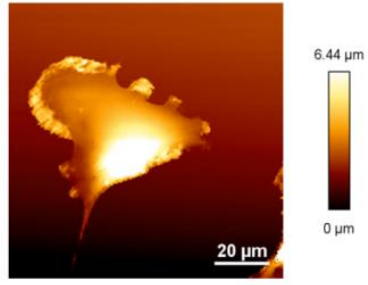

(d)

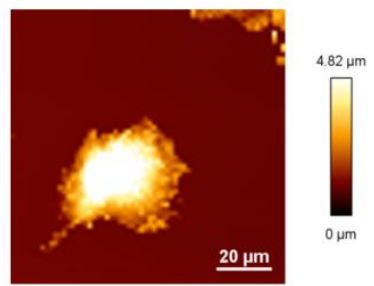

(h)

Fig. 9. AFM contact point images extracted from QI mode $\circledR$ images of GBM cells: (a-d) SNB-19 cells and (e-h) UP-007 cells.

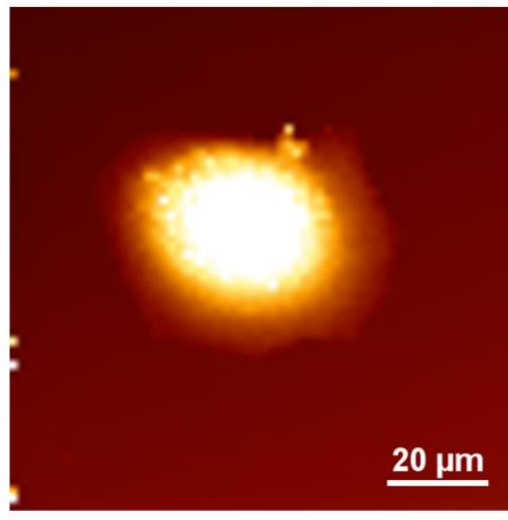

(a)

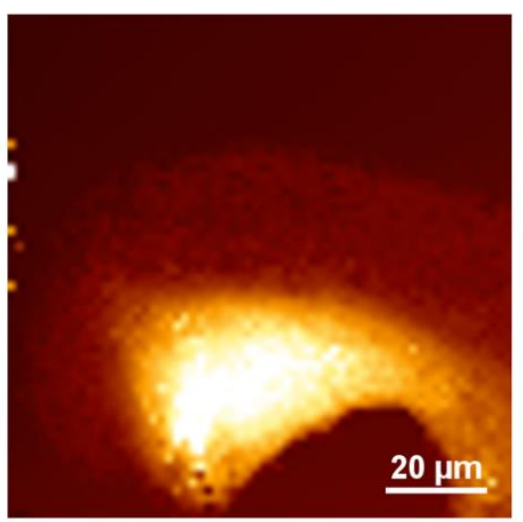

(C)
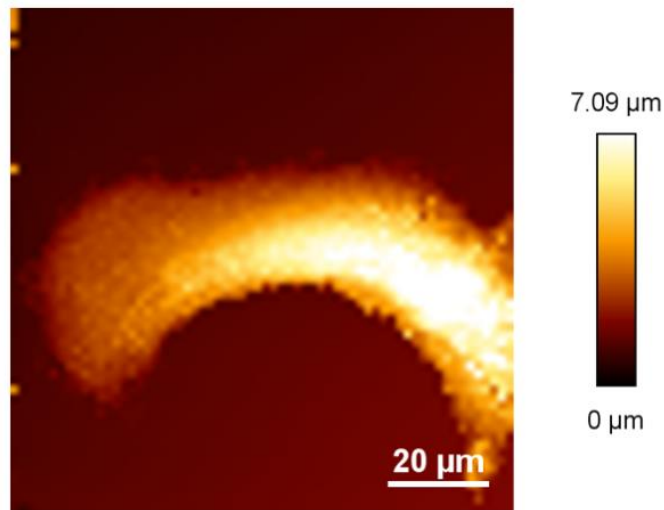

$0 \mu \mathrm{m}$

(b)

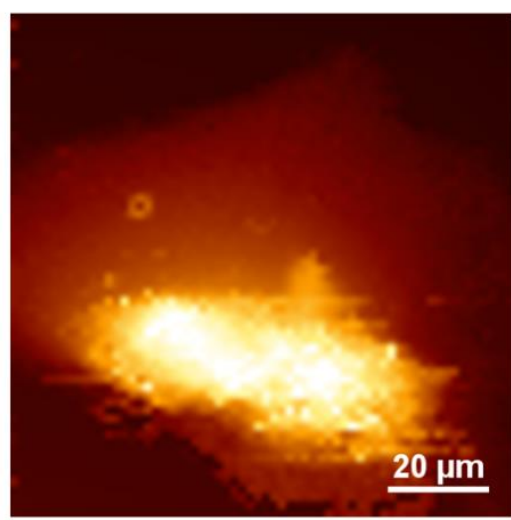

$13.4 \mu \mathrm{m}$

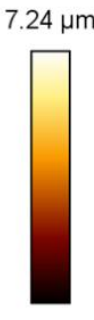

$0 \mu \mathrm{m}$

(d) 
BioNanoScience

First online: 06 January 2016, pp. 1-7, doi: 10.1007/s12668-015-0188-3

www.link.springer.com/article/10.1007/s/12668-015-3

Fig. 10. AFM contact point images extracted from QI mode ${ }^{\circledR}$ images of low-grade (WHO grade I) glioma cells.

A statistical comparison of the lamellipodia heights for the different cell lines is summarised in Fig. 11. The two astrocyte cell lines showed no difference in periphery cell heights $(p>0.05)$ and had the lowest values. The lamellipodia heights in SNB-19 and UP-007 cells were both greater than those of the astrocytes $(p<0.0001$, except between CC-2565 and UP-007, $p<0.001$ ). These results suggest that some elaborate concentration of cytoskeletal structures, probably F-actin, may explain the large height values of the lamellipodia. Interestingly, a difference in lamellipodia heights were noted between the two types of gliomblastoma cell lines $(p<0.0001)$, possibly reflecting the degree of invasive potential between these two cells lines as evidenced in the invasion assay. The low-grade glioma cell line had lamellipodia heights similar to the astrocytes (both cell types; $p>0.05)$, although were less than those of the gliomas $(p<0.0001$ and $p<0.01$ for SNB-19 and UP-007, respectively). The low-grade SEBTA-048 cells would be expected to be less invasive than the WHO grade IV gliomas, although this was not investigated.

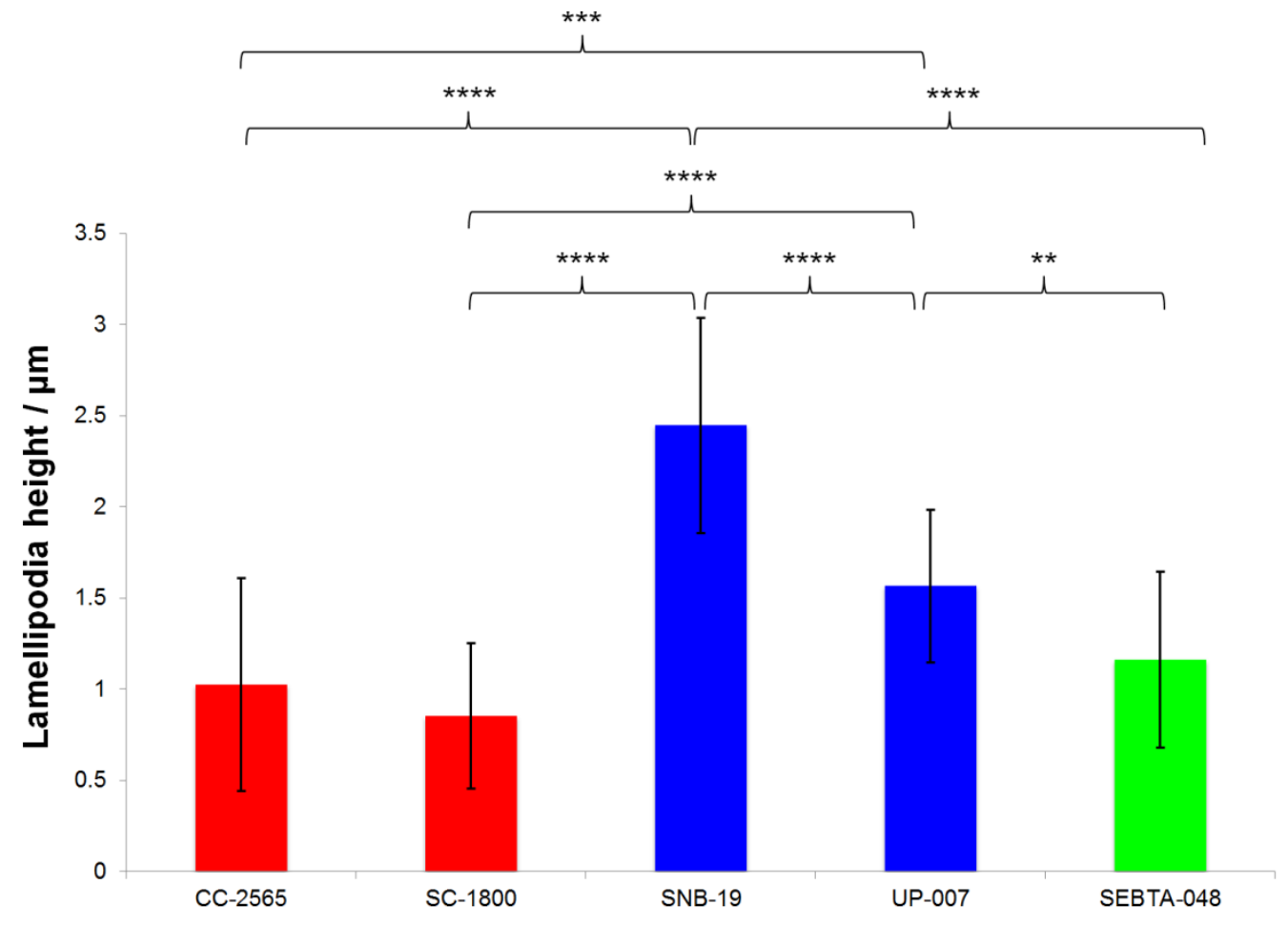

Cell line

Fig. 11. Lamellipodia heights measured from AFM contact point images of the various cell lines; CC-2565 and SC-1800 (3 cells per cell line), SNB-19, UP-007 and SEBTA-048 (4 cells per cell line); 10 measurements per cell; $*, p<0.05 ; * *, p<0.01 ; * * *, p<0.001 ; * * * *, p<0.0001$; red = non-neoplastic astrocytes, blue = glioblastoma high-grade (WHO grade IV), green = low-grade (WHO grade I) glioma cell line.

To our knowledge, these are the first AFM images that show a heightened edge at cell peripheries. Numerous AFM studies show cell topography to slope away from the nucleus gradually to the cell margin and do not report the raised topography at cell edges, both for glioma cells [17-20] and other cells (cancerous and non-cancerous) [21-23]. Staining 
of F-actin, intermediate filament and microtubules in these cells show fluorescence at the cell periphery, but also throughout the cytoplasm [7] (although largely around the nuclear regions, in the case of vimentin [8]. Therefore, some elaborate concentration of cytoskeletal structures, possibly F-actin, may explain the large height values of the lamellipodia. The size of the heightened lamellipodia in high-grade glioma cells over astrocytes and low-grade glioma cells may provide new insights into controlling cellular invasion.

\section{Conclusions}

For the first time, AFM has been used to measure the lamellipodia heights of high-grade glioma cells and these have been found to be higher than non-neoplastic astrocyte control cells. Equivalent heights of low-grade glioma cells were found to be identical to those of the astrocytes and less than those of the GBMs, although future studies should incorporate more low-grade glioma cell lines. These results suggest that lamellipodia height could be used to monitor and control cell invasion in brain tumours.

Acknowledgements We thank Drs Robert Field and Alex Winkle from JPK Instruments, Cambridge, UK for loan of the NanoWizard 3 AFM instrument, and Brain Tumour Research for support. We also thank Prof Keyoumars Ashkan and Dr Stavros Polyzoidis, of the Department of Neurosurgery, King's College Hospital, London, UK, for providing the brain tumour biopsy sample for establishment as a primary cell line (SEBTA-048).

\section{Compliance with Ethical Standards}

Ethics Statement All cell lines established 'in house' were conducted in accordance with the National Research Ethics Service (NRES) instructions and under Ethics permission $11 / \mathrm{SC} / 0048$.

\section{References}

1. Louis DN, Ohgaki H, Wiestler OD, Cavenee WK, Burger PC, Jouvet A, Scheithauer BW, Kleihues P (2007) The 2007 WHO Classification of tumours of the central nervous system. Acta Neuropathol 114: 97-109. doi: 10.1007/s00401-007-0243-4

2. Hoa VKY, Reijneveld JC, Enting RH, Bienfait HP, Robe P, Baumert BG, Visser O (2014) Changing incidence and improved survival of gliomas. Eur J Cancer 50: 2309-2318. doi: 10.1016/j.ejca.2014.05.019

3. Cross SE, Jin YS, Rao J, Gimzewski JK (2007) Nanomechanical analysis of cells from cancer patients. Nat Nanotechnol 2: 780-783. doi: 10.1038/nnano.2007.388

4. Docheva D, Padula D, Schieker M, Clausen-Schaumann H (2010) Effect of collagen I and fibronectin on the adhesion, elasticity and cytoskeletal organization of prostate cancer cells. Biochem Biophys Res Commun 402: 361-366. doi:10.1016/j.bbrc.2010.10.034

5. Wang B, Lancon P, Bienvenu C, Vierling P, Di Giorgio C, Bossis G (2013) A general approach for the microrheology of cancer cells by atomic force microscopy. Micron 44: 287-297. doi: 10.1016/j.micron.2012.07.006

6. Vadillo-Rodriguez V, Dutcher JR (2009) Dynamic viscoelastic behavior of individual Gramnegative bacterial cells. Soft Matter 5: 5012-5019. doi: 10.1039/b912227c

7. Maherally Z, Smith JR, An Q, Pilkington GJ (2012) Receptors for hyaluronic acid and poliovirus: A combinatorial role in glioma invasion? PLoS One 7: e30691. doi: 10.1371/journal.pone.0030691 
BioNanoScience

First online: 06 January 2016, pp. 1-7, doi: 10.1007/s12668-015-0188-3

www.link.springer.com/article/10.1007/s/12668-015-3

8. Maherally Z, Smith JR, Ghoneim MK, Dickson L, An Q, Fillmore HL, Pilkington GJ (2015) Silencing of CD44 in glioma leads to changes in cytoskeletal protein expression and cellular biomechanical deformation properties as measured by AFM nanoindentation. BioNanoScience 1-11. doi:10.1007/s/12668-015-0189-2

9. Romet-Lemonne G, Jégou A (2013) Mechanotransduction down to individual actin filaments. Eur J Cell Biol 92: 333-338. doi: 10.1016/j.ejcb.2013.10.011

10. Ridley AJ (2011) Life at the leading edge. Cell 145: 1012-1022. doi: 10.1016/j.cell.2011.06.010

11. Svitkina TM, Borisy GG (1999) Arp2/3 complex and actin depolymerizing factor/cofilin in dendritic organization and treadmilling of actin filament array in lamellipodia. J Cell Biol 145: 1009-1026. doi: $10.1083 / j c b .145 .5 .1009$

12. Yamada $H$, Ade $T$, Li S-A, Masuoka $Y$, Isoda $M$, Watanabe $M$, Nasu $Y$, Kumon $H$, Asai A, Takei $K$ (2015) Dynasore, a dynamic inhibitor, suppresses lamellipodia formation and cancer cell invasion by destabilizing actin filaments. Biochem Biophys Res Commun 390: 1142-1148. doi:10.1016/j.bbrc.2009.10.105

13. Chopinet L, Formosa C, Rols MP, Duval RE, Dague E (2013) Imaging living cells surface and quantifying its properties at high resolution using AFM in $\mathrm{QI}^{\mathrm{TM}}$ mode. Micron 48: 26-33. doi: 10.1016/j.micron.2013.02.003

14. An Q, Fillmore HL, Vouri M, Pilkington GJ (2014) Brain tumour cell line authentication, an efficient alternative to capillary electrophoresis by using a microfluidics-based system. Neurooncology 16: 265-273. doi: 10.1093/neuonc/not202

15. Pilkington GJ, Akinwunmi J, Ognjenovic N, Rogers JP (1993) Differential binding of anti-CD44 on human gliomas in vitro. Neuroreport 4: 259-62. doi: 10.1097/00001756-199303000-00008

16. Hutter JL, Bechhoefer J (1993) Calibration of atomic-force microscope tips. Rev Sci Instrum 64: 1868-1873. doi: $10.1063 / 1.1143970$

17. Fillmore HL, Chasiotis I, Cho SW, Gillies GT (2003) Atomic force microscopy observations of tumour cell invadopodia: novel cellular nanomorphologies on collagen substrates. Nanotechnology 14: 73-76. doi: 10.1088/0957-4484/14/1/317

18. Chasiotis I, Fillmore HL, Gillies GT (2003) Atomic force microscopy measurement of cytostructural elements involved in the nanodynamics of tumour cell invasion. Nanotechnology 14 : 557-561. doi: 10.1088/0957-4484/14/5/314

19. D'Agostino DP, Olson JE, Dean JB (2009) Acute hyperoxia increases lipid peroxidation and induces plasma membrane blebbing in human U87 glioblastoma cells. Neuroscience 159: 10111022. doi:10.1016/j.neuroscience.2009.01.062

20. Selmeczi D, Szabo B, Sajo-Bohus L, Rozlosnik N (2001) Morphological changes in living cell cultures following $\alpha$-particle irradiation studied by optical and atomic force microscopy. Radiat Measur 34: 549-553. doi: 10.1016/S1350-4487(01)00226-8

21. Bastatas L, Martinez-Marin D, Matthews J, Hashem J, Lee YJ, Sennoune S, Filleur S, MartinezZaguilan R, Park S (2012) AFM nano-mechanics and calcium dynamics of prostate cancer cells with distinct metastatic potential. Biochim Biophys Acta 1820: 1111-1120. doi: 10.1016/j.bbagen.2012.02.006

22. Zhang $X$, Tang Q, Wu L, Huang J, Chen $Y$ (2015) AFM visualization of cortical filaments/network under cell-bound membrane vesicles. Biochim Biophys Acta 1848: 2225-2232. doi: $10.1016 /$ j.bbamem.2015.06.025

23. Birukova AA, Arce FT, Moldobaeva N, Dudek SM, Garcia JGN, Lal R, Birukov KG (2009) Endothelial permeability is controlled by spatially defined cytoskeletal mechanics: Atomic force microscopy force mapping of pulmonary endothelial monolayer. Nanomed Nanotechnol Biol Med 5: 30-41. doi: 10.1016/j.nano.2008.07.002 